The Israeli Journal of Aquaculture - Bamidgeh, IJA_73.2021.1237639, 9 pages

CCBY-NC-ND-4.0 • https://doi.org/10.46989/001c.21213

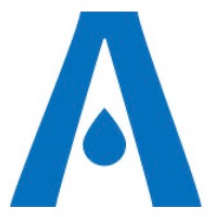

The IJA is a peer-reviewed open-access, electronic journal, freely available without charge to users

Produced by the AquacultureHub non-profit Foundation Sale of $I J A$ papers is strictly forbidden

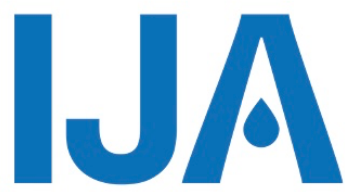

\title{
Changes in juvenile sturgeon erythrocytes across age classes reflects evolutionary development
}

\author{
Shiyong Yang ${ }^{1,}$, Chaoyang Zhang ${ }^{1}$, , Quan Gong ${ }^{2}$, Wenqiang $\mathrm{Xu}^{1}$, Datian \\ $\mathrm{Li}^{1}$, Zhao Liu ${ }^{2}$, Jiayun $\mathrm{Wu}^{3}$, Liangyu $\mathrm{Li}^{3}$, Jun $\mathrm{Du}^{2,}{ }^{*}$, Xiaoli Huang ${ }^{1, *}$ \\ ${ }^{1}$ Department of Aquaculture, Sichuan Agricultural University, Chengdu, Sichuan 611130, \\ China \\ ${ }^{2}$ Fisheries Research Institute, Sichuan Academy of Agricultural Sciences, Chengdu \\ 611731, China \\ ${ }^{3}$ Fisheries Research, Chengdu Academy of Agricultural and Forestry Sciences, Chengdu, \\ Sichuan 611130, China
}

Key words: Sturgeon, erythrocytes, morphology, development

\begin{abstract}
Erythrocytes are important for gas transport and exchange in animals and play an important role in maintaining normal functions of the body. The purpose of this study was to observe the changes in erythrocyte morphology during the development of juvenile sturgeon (Acipenser baeri $q \times$ Acipenser schrenckii $\hat{o}$ hybrid F1) and to describe the relationship between erythrocyte morphology and development. We compared the erythrocyte morphology of juvenile sturgeon in three developmental stages. The results showed that the blood index of the sturgeon in different age classes was significantly different. The erythrocyte volume in the blood became smaller and the shape of erythrocytes changed from circular to elliptical. With the increase in age, the number of selfdividing erythrocytes, and the types and numbers of organelles in the erythrocytes, decreased. The results demonstrated that the morphology and the internal structure of erythrocytes develop to be more beneficial for gas transport reflecting the needs for juvenile sturgeon maintenance and environmental changes.
\end{abstract}

\footnotetext{
* Corresponding author, Email: hxlscau@126.com (X.L. HUANG); dujun9100@126.com (J. DU).
} The first three authors contributed the work equally. 


\section{Introduction}

Erythrocytes are critical for gas transport and exchange in animals and play an important role in maintaining normal functions of the body (Rowley, 1995). In the broadest evolutionary sense of animals, as habitats changed from aquatic to terrestrial, the erythrocytes of animals underwent corresponding changes (Qing et al., 2011). In terms of overall animal hematology studies, the more evolved the animal, the smaller and more numerous the erythrocytes tend to be, as to obtain greater gas transport capacity (Campbell, 2015). The emergence of vertebrates allowed the differentiation of erythrocytes from blood cells, specifically for gas exchange. Earlier studies have shown that changes in genome size in vertebrates are usually positively correlated with the size of erythrocytes, and decrease in turn in fish, amphibians, reptiles, birds, and mammals (Commoner et al., 1964). The self-dividing function of erythrocytes produced by the hypoplastic hematopoietic organs during animal evolution is widely found in fish blood (Cao et al., 2005; $\mathrm{Li}$ et al., 2011). The hematopoietic organs of higher animals are fully developed, the erythrocytes are highly differentiated, and cell functions are maximized (Harvey et al., 1977). Although research on erythrocytes is extensive (Bytyutskyy et al., 2014), the changes in erythrocytes at different ages within a species is less clear.

Sturgeon, an ancient fish, has an evolutionary history that spans the evolutionary periods from lower to higher animals (Howes, 1991). Through 200 million years, it is not clear whether the erythrocytes have changed during growth and development. However, hematological studies have shown that sturgeon have a rich variety of blood cells, including many types of erythrocytes (Ya et al., 2017; Zexia et al., 2007). In the actual breeding process, it was found that the blood color of sturgeon changes significantly during the juvenile stage. Therefore, it is likely that changes in erythrocytes in response to the body's needs can be observed in sturgeon blood.

The purpose of this study was to understand the changes of erythrocyte composition and morphology in juvenile sturgeon at different ages. We evaluated erythrocytes and related hematological indicators at different ages of juvenile sturgeon. Also, the morphology and ultrastructure of the erythrocytes were investigated. The results provide information on potential coordinated changes between erythrocytes and the growth of sturgeon, perhaps reflecting evolutionary trajectories in the lineage.

\section{Fish maintenance and treatment protocol}

Three age classes of 50 juvenile sturgeons (Acipenser baeri $q \times$ Acipenser schrenckii $\hat{o}$ hybrid F1), 40, 180, and 540 days, were purchased from Sichuan Runzhao Fishery Co., Ltd. China, respectively. The average body weight was $9.78 \pm 1.90 \mathrm{~g}, 82.8 \pm 10.37 \mathrm{~g}$ and $624.35 \pm 27.11 \mathrm{~g}$; the average body length was $10.51 \pm 1.23 \mathrm{~cm}, 22.22 \pm 1.11 \mathrm{~cm}$ and $45.83 \pm 1.54 \mathrm{~cm}$, respectively. Fish were housed based on the three age groups and acclimatized in the laboratory for 2 weeks before experimentation. Fish were exposed to a light/dark cycle of 12:12, and uninterrupted oxygen was supplied to ensure more than 7.8 $\mathrm{mg} / \mathrm{L}$ dissolved oxygen; a $\mathrm{pH}$ of 7.0-8.0 was maintained, as was ammonia nitrogen and nitrite at 0-0.02 $\mathrm{mg} / \mathrm{L}$. Subsequently, the fish were anesthetized using an MS-222 buffer and the blood was collected from the tail vein. All animal handling procedures were approved by the animal care and use committee of Sichuan Agricultural University following the animal experiment guidelines of Sichuan Agricultural University, License No. DYS20144657.

$R B C, H b$, and TP content examination

The blood samples collected from each group were added into $0.65 \% \mathrm{NaCl}$ buffer to prepare a red blood cell $(\mathrm{RBC})$ dilution $(\times 200)$, and the number of RBC were counted with a blood count board (FK-KN253-267, Corning, USA) described by Yuan et al (Yuan et al., 2006). The hemoglobin ( $\mathrm{Hb}$ ) and total plasma (TP) content of the blood were determined with $\mathrm{Hb}$ diluent kit (C021, Nanjing Jiancheng, China) and BCA protein quantitative checkerboard kit (A045-4, Nanjing Jiancheng, China) following the instruction manual.

Erythrocyte size measurements 
A $0.2 \mathrm{~mL}$ mixed sample of blood from three groups $(\mathrm{N}=9)$ was diluted with a $0.65 \%$ $\mathrm{NaCl}$ solution to prepare a blood smear $(\mathrm{N}=20)$ and then stained with a fast Wright stain solution (D007, Nanjing Jiancheng, China). Three suitable fields were chosen for observation on each smear and five erythrocytes were selected randomly for measurement. Slides were observed and photographed using an optical microscope (Nikon, Tokyo, Japan). Adobe Photoshop CS6 (64 Bit) was used to measure the length and diameter of erythrocytes and nuclei.

Flow Cytometry Assay

A flow cytometry assay was performed as described previously (Chen et al., 2011). A $20 \mu \mathrm{L}$ mixed sample of the blood was taken from three groups $(\mathrm{N}=9)$, and a single-cell suspension of blood cells was prepared with a concentration of $1 \times 106$ cells $/ \mathrm{mL}$. Then $1 \mathrm{~mL}$ cell suspension was transferred into a $5-\mathrm{mL}$ culture tube and centrifuged at $250 \times \mathrm{g}$ for 4 min. Subsequently, the cell suspension was permeabilized with $500 \mu \mathrm{L} 0.25 \%$ Triton X-100 for $10 \mathrm{~min}$ at $4^{\circ} \mathrm{C}$, and incubated with $50 \mathrm{uL}$ PI/RNase Staining Buffer (550825, BD, USA) for 30 min at $4^{\circ} \mathrm{C}$ in a darkroom. A $400 \mu \mathrm{L}$ sample of PBS ( $\left.\mathrm{pH} 7.2-7.6\right)$ was added and the cell cycle phase distribution was tested by flow cytometry (CytoFLEX, Backman, USA) within $45 \mathrm{~min}$ and analyzed by FlowJo 7.6 software. The proliferating index value was calculated by the following formula: PI $=(\mathrm{S}+\mathrm{G} 2 \mathrm{M}) /(\mathrm{G} 0 \mathrm{G} 1+\mathrm{S}+\mathrm{G} 2 \mathrm{M}) \times 100 \%(\mathrm{G} 0 / \mathrm{G} 1$ represents the DNA content of diploid cells; G2M represents the DNA content of tetraploid cells; and S represents the DNA content between diploid and tetraploid cells).

Ultrastructural examination

The mixed blood $(0.2 \mathrm{~mL})$ from each group $(\mathrm{N}=9)$ was centrifuged and the upper plasma discarded. The blood cells were immediately fixed in $2.5 \%$ glutaraldehyde and postfixed in $1 \%$ osmium $0.1 \mathrm{M}$ phosphate PBS buffer ( $\mathrm{pH} 7.4)$. After dehydration in graded alcohol, the samples were embedded in Araldite. The blocks were sectioned on a microtome with a glass knife. The sections $(6.575 \mu \mathrm{m}$ thick) were placed on uncoated copper grids, stained with uranyl acetate, and post-stained with $0.2 \%$ lead citrate. A transmission electron microscope (HT7700, HITACHI, Japan) was used to observe and collect images for analysis. Another $0.2 \mathrm{~mL}$ of the blood sample was repeated for the centrifugation, fixation, and dehydration procedures. The subcellular structures of the erythrocytes were examined with a scanning electron microscope (SU8100, HITACHI, Japan).

Statistical analysis

The normality of the data distributions was checked using a Shapiro-Wilk's test, and the assumption of homoscedasticity of variances was tested using Levene's test. Data were expressed as mean \pm SD of the number of animals used in each group. Statistical analysis was performed using a one-way ANOVA and Tukey posthoc tests in SPSS 22.0. $P<0.05$ indicated a significant difference between groups.

\section{Results}

Significant differences in blood parameters in different groups

Larger animals require more oxygen and cellular fuel to carry out respiration than smaller animals (Glencross et al., 2006). Our results showed that the number of RBC increased with age. The number in the 540-d fish was more than 3 times compared with that in $40 \mathrm{~d}$ and $180 \mathrm{~d}$ fish (Figure 1A; $P<0.05$ ). The $\mathrm{Hb}$ content increased with age and there were significant differences among age stages (Figure 1B; $P<0.05$ ). Also, the TP content increased with time and the values at $180 \mathrm{~d}$ and $450 \mathrm{~d}$ were significantly different compared with that at $40 \mathrm{~d}$ (Figure 1C). 

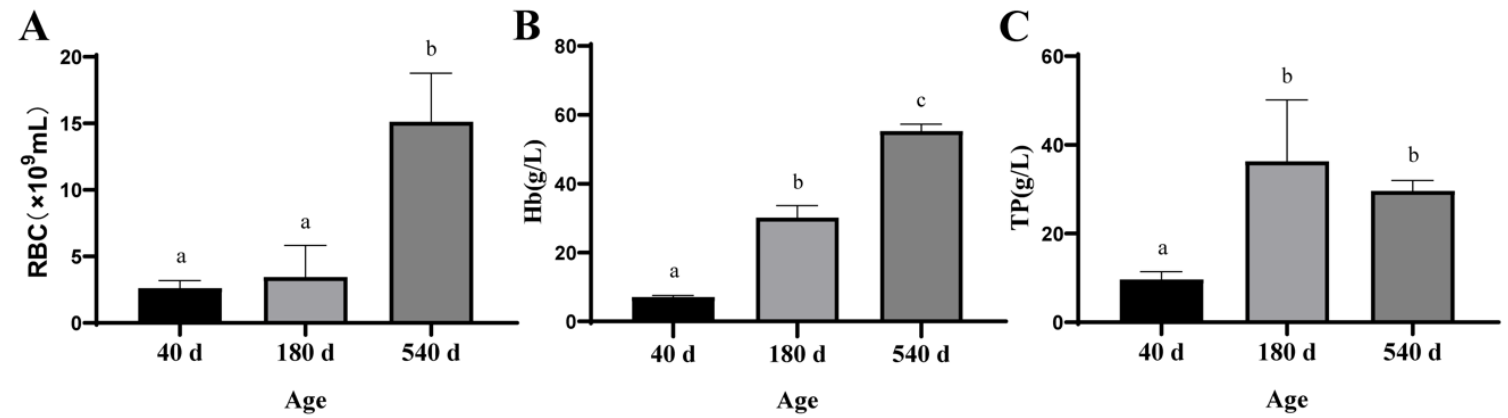

Figure 1 The content of RBC (Panel A), $\mathrm{Hb}(B)$, and TP (C) in the blood for different age groups. Different letters indicate a significant difference among different ages $(P<0.05)$. RBC: red blood cells; Hb: hemoglobin; TP: total plasma protein. Values are mean $\pm S D, n=5$.

\section{Erythrocyte size and shape}

Studies have shown that the oxygen-carrying capacity of erythrocytes is related to their volume and morphology (Dunaway et al., 1965). It was found in this study that in older age classes, the diameter of erythrocytes decreased (Figure 2A). As shown in Figure 2C, the longest/shortest diameter ratio of erythrocytes increased with age and 180 d was significantly different from $40 \mathrm{~d}$. The same trend was found in the nucleus that showed a trend of decreasing the longest and shortest diameters (Figure 2B). The nuclear long/short diameter ratio was highest at $180 \mathrm{~d}$ (Figure 2D).
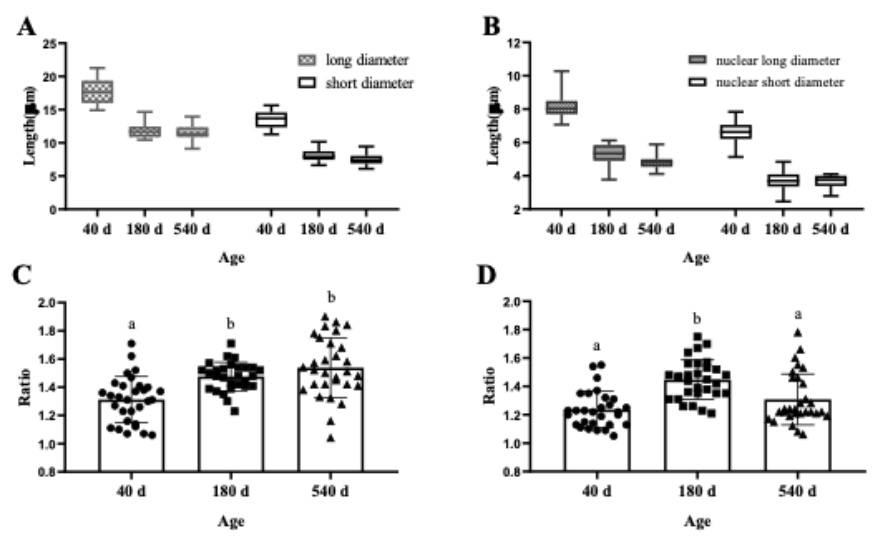

Figure 2 The size and shape of erythrocytes at different ages. A-B: Comparison of the diameters of erythrocytes and erythrocyte nuclei at different ages. C-D: Comparison of erythrocyte long/short diameter ratio and nuclear longest/shortest diameter ratio at different ages. The different letters indicate significant differences among different ages $(P<0.05)$. Values are mean $\pm \mathrm{SD}, \mathrm{n}=30$.

\section{Self-dividing ability of erythrocytes}

G0/G1, G2M, and S phase were used to represent the DNA content of diploid cells, tetraploid cells, and those cells in which DNA content was between diploid and tetraploid cells, respectively. The higher the PI value, which reflects the sum of G2M and S phase values, the faster the cell division and proliferation. The ratio of blood cell cycles and cell morphology at different ages is shown in Figure 3A - F. For the 540-d group, the percentage of cells in the G0G1 phase was higher than that of $40 \mathrm{~d}$ and $180 \mathrm{~d}$, but the PI decreases after $40 \mathrm{~d}$ and $180 \mathrm{~d}(P<0.05)$ (Figure 3G). The results demonstrated that the proliferation capacity of blood cells decreases as sturgeon grow. 

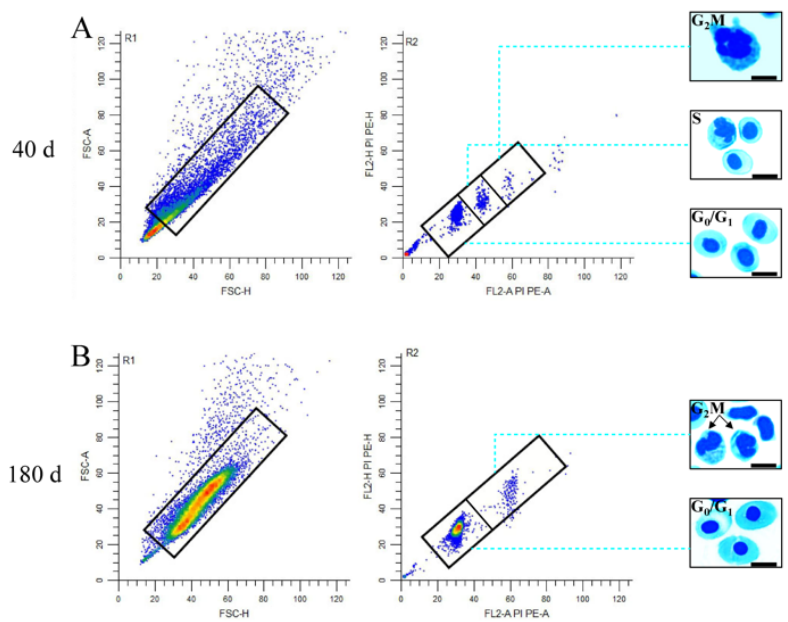

$\mathrm{D}_{3}$

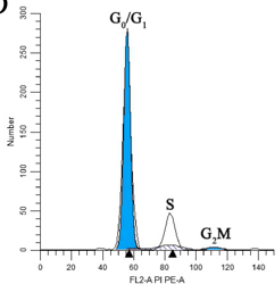

$\mathrm{E}_{\text {ฐ }}$
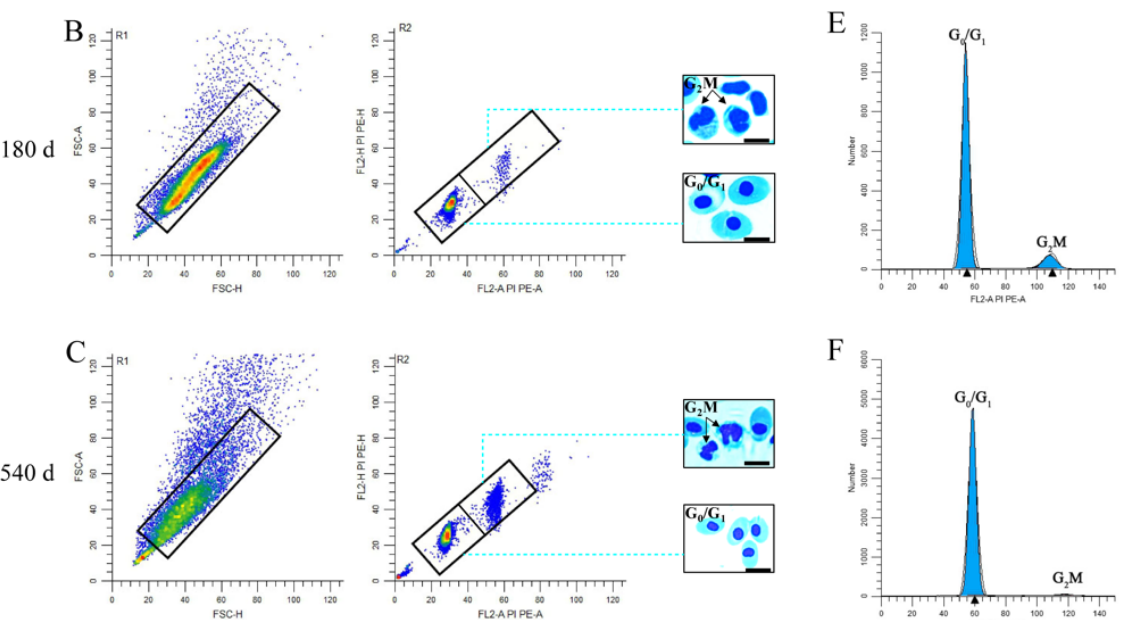

G

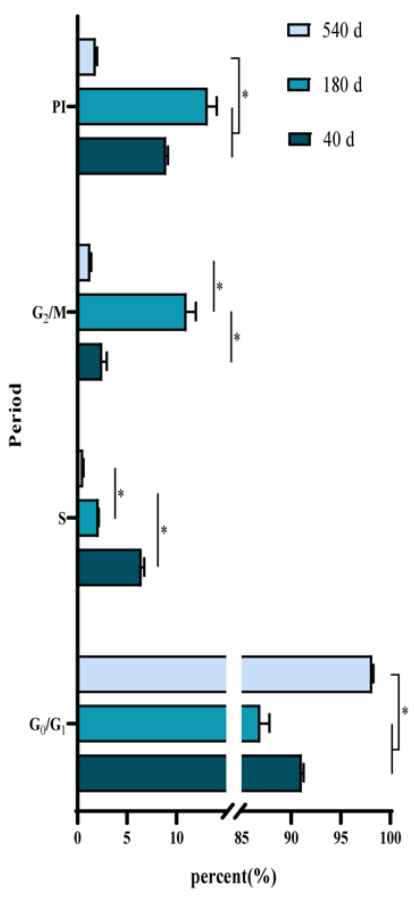

Figure 3 The proliferation of blood cells in different age stages. A-C: The morphology of blood cells in each cell cycle at different ages. D-F: The proportions of each cell cycle at $40 \mathrm{~d}, 180 \mathrm{~d}$, and 540 d. G: The ratio of the blood cell cycle groups at different ages. A "*" represents a significant difference between age groups $(P<0.05)$.

\section{Ultrastructural characteristics of erythrocytes}

The results showed that the surface of erythrocytes in the youngest age group was relatively rough and inlaid with a large number of concave holes. However, with an increase in age, the surface of the erythrocytes tended to be smoother (Figure 4A). Image] v. 1.50 was used to count the number of the concave holes of erythrocyte surfaces in each group. Fewer concave holes were found on the surface of erythrocytes at older ages, and the 540 group was significantly different from that at $40 \mathrm{~d}$ and $180 \mathrm{~d}(P<0.05)$ (Figure 4B).

Transmission electron microscopy revealed mitochondria and a small number of lysosomes in the erythrocyte cytoplasm of the sturgeon at $40 \mathrm{~d}$. The mitochondria were of two types: small mitochondria M1 and large mitochondria M2. However, the number of organelles in erythrocytes gradually decreased with age and disappeared completely at $540 \mathrm{~d}$ (Figure 4C-D). 

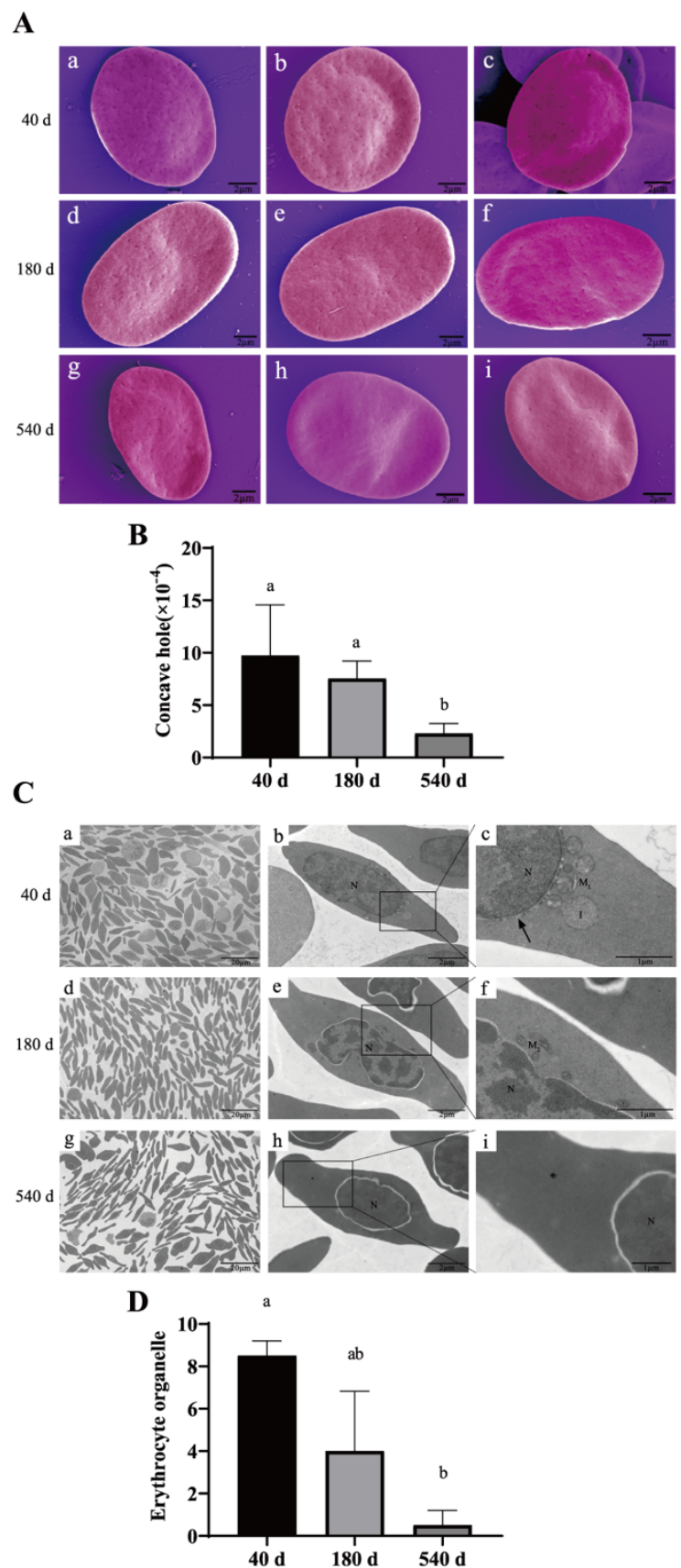

Figure 4 Ultrastructural observations of erythrocytes at different ages. A: Scanning electron microscopy of erythrocytes at different ages (a-c, $40 \mathrm{~d} ; \mathrm{d}-\mathrm{f}, 180 \mathrm{~d} ; \mathrm{g}-\mathrm{i}, 540 \mathrm{~d}$ ). Adobe Photoshop CS6 (64 Bit) was used for pseudo-coloring. B: The concave hole number in erythrocyte membranes at different ages. C: Transmission electron microscopy of erythrocytes at different ages (a-c, $40 \mathrm{~d}$; df, $180 \mathrm{~d} ; \mathrm{g}-\mathrm{i}, 540 \mathrm{~d}$ ). D: The number of organelles at different ages. N: nucleus of erythrocyte; M1: small mitochondria. M2: large mitochondria; I: lysosome; "๕" nuclear membrane. The different letters in the graphs indicate a significant difference among different age groups $(P<0.05)$.

\section{Discussion}

Erythrocytes are critical for gas transport and exchange in animals and play an important role in maintaining normal functions of the body (Rowley, 1995). Studies have shown that there were significant differences in the morphology and composition of erythrocytes based on animal evolution patterns (Campbell, 2015; Hawkey et al., 1991; Qing et al., 2011). The surface morphology of erythrocytes in different species also varies. Erythrocytes are not well-differentiated in the hemolymph of oyster (e.g., Crassostrea 
madrasensis) (Ittoop et al., 2006), whereas amphibians (e.g., Hoplobatrachus chinensis and Lithobates catesbeiana) and reptiles (e.g., Trionyx sinensis) have evolved erythrocytes which have surface protrusions and shallow caves (Qing et al., 2011). Mammals' mature erythrocytes have smooth membranes and an oval morphology to facilitate movement in the capillaries (Elgsaeter A and Mikkelsen A. 1991). Previous research has demonstrated that the structural characteristics of the surface of erythrocytes are closely related to the gas exchange capacity, and the number of erythrocyte membrane concave holes is positively correlated with the gas exchange capacity (Chiancone et al., 2000; Gambacurta. et al., 1993; Qing et al., 2011). It implied that in the evolution from mollusks to mammals, erythrocyte morphology tends to be smaller and more elliptical, and the surface of the cell membrane tends to be smoother. However, it is unclear whether this change could occur within a species at different stages of development.

Therefore, sturgeon, a cartilaginous fish that has existed on Earth for 200 million years, was used to examine possible ontogenetic changes. The above results show that the number, shape, and components of erythrocytes changed with the age of the juvenile sturgeon. We observed hole-like caves on the surface of the erythrocyte membranes for the three age classes, with the number decreasing with age. The morphology of the erythrocyte membrane of the $540 \mathrm{~d}$ fish was somewhat similar to that of higher mammals. The caves found in the erythrocyte membranes of juvenile sturgeon might be used to compensate for the inefficiency of gas exchange in early life stages.

Changes in the types and number of organelles also reflect changes in the demand of erythrocytes for oxygen in the body. Studies have found that the erythrocyte cytoplasm of mollusks and crustaceans usually contain basophilic and eosinophilic particles that some researchers believe are lysosomes (Kuchel et al., 2010). However, there are no organelles in the erythrocyte cytoplasm of mature mammal lineages (Martina et al., 2017). Researchers have shown that there are no classified erythrocytes in the hemolymph of mollusks, whose blood cells perform functions other than oxygen transport, and the particles in the cytoplasm are considered lysosomes (Kuchel et al., 2010). The erythrocyte cytoplasm of fish (e.g., Carassius auratus), amphibians (e.g., Lithobates catesbeiana), and reptiles (e.g., Trionyx sinensis) have no obvious organelles (Qing et al., 2011). For most mammals, the erythrocytes contain more $\mathrm{Hb}$ and almost no organelles (Martina et al., 2017; Orci et al., 1975). Studies have shown that organelles in fish erythrocytes play an important role in the synthesis of $\mathrm{Hb}$ and assisting the body in aerobic respiration (Murad et al., 1993). In the present study, we found a large number of mitochondria and lysosomes existed in the erythrocytes of the younger age group (40 d), but they decrease with age. This suggests that, through millions of years of environmental selection, sturgeon had evolved an appropriate "age-stage blood system" that makes the most effective internal changes (e.g., changes in erythrocytes) in response to body and external changes (Roger et al., 2002). Erythrocytes take part in immune function during dysgenesis in the early life stages of sturgeon. Conversely, they are more dedicated to oxygen transport with different morphology and structures at older life stages. The above research provided some theoretical conditions for the sturgeon to survive on earth for hundred million years.

\section{Acknowledgements}

We thank all authors for stimulating discussions and support. All authors read and approved the final manuscript. We thank International Science Editing (http://www.internationalscienceediting.com) for editing this manuscript. This research was supported by Applied Basic Research from Technological Office of Sichuan Province (2015JY0206); Applied Basic Research from Technological Office of Sichuan Province (2019YJ0515); Supported by the Project of Provincial 13th Five Years' Animal Breeding of Sichuan Province (2016NYZ0047).

\section{References}

Bytyutskyy D., Kholodnyy V. and Martin F., 2014. 3-D structure, volume, and DNA content of erythrocyte nuclei of polyploid fish. Cell Biology International, 38 (6): 708-715. https://doi.org/10.1016/0304-4157(91)90017-Q 
Campbell TW., 2015. Exotic Animal Hematology and Cytology. Colorado State University, America.

Cao FJ., Liu CW. and Li CL., 2005. Microstructure of Peripheral Blood in Plectorhinchus cinctus. Acta Laser Biology Sinica, 10 (3):181-186. https://10.1563/0-742.1

Chen T., Cui H., Cui Y., Bai C. and Gong T., 2011. Cell-cycle blockage associated with increased apoptotic cells in the thymus of chickens fed on diets high in fluorine. Human and Experimental Toxicology, 30 (7): 685-692. https:// doi.org/10.1177/0960327110379022

Chiancone E. and Boffi A, 2000. Structural and thermodynamic aspects of cooperativity in the homodimeric hemoglobin from Scapharca inaequivalvis. Biophysical Chemistry, 86 (2-3): 173-178. https:// doi.org/10.1016/s0301-4622(00)00162-9

Commoner B., 1964. Roles of Deoxyribonucleic Acid in Inheritance. Nature, 202 (4936): 960-968.

Dunaway PB. and Lewis LL, 1965. Taxonomic Relation of Erythrocyte Count, Mean Corpuscular Volume, and Body-Weight in Mammals. Nature, 205 (4970): 481-484. https:// doi.org/10.1038/205481a0

Elgsaeter A. and Mikkelsen A, 1991 Shapes and shape changes in vitro in normal red blood cells. Biochimica et Biophysica Acta 1071 (3):273-290. https://doi.org/10.1016/0304-4157(91)90017-Q

Gambacurta A., CristinaPiro M. and Ascoli F, 1993. Cooperative homodimeric hemoglobin from Scapharca inaequivalvis. cDNA cloning and expression of the fully functional protein in E. coli. Federation of European Biochemical Societies Letters, 330 (1): 90-94. https://doi.org/10.1016/0014-5793(93)80926-L

Glencross B. and Felsing M, 2006. Influence of fish size and water temperature on the metabolic demand for oxygen by barramundi, Lates calcarifer(Bloch), in freshwater. Aquaculture Research, 37 (11): 1055-1062. https://doi.org/10.1016/00145793(93)80926-L

Harvey JW. and Kaneko JJ, 1977. Mammalian Erythrocyte Metabolism and Oxidant Drugs. Toxicology and Applied Pharmacology, 42 (2): 253-261. https:// doi.org/10.1016/0041-008X(77)90002-3

Hawkey CM., Bennett PM., Gascoyne SC., Hart MG. and Kirkwood JK., 1991. Erythrocyte size, number and haemoglobin content in vertebrates. British Journal of Haematology, 77 (3): 392-397. https://doi.org/10.1111/j.1365-2141.1991.tb08590.x

Howes GJ., 1991. Systematics and biogeography: an overview. Springer Netherlands.

Ittoop G., George KC., Sanil NK., George RM. and Nisha PC., 2006. Characterization of haemocytes of the Indian edible oyster, Crassostrea madrasensis (Preston). Aquaculture Research, 37 (16): 1636-1643. https://doi.org/10.1111/j.1365-2109.2006.01606.x

Kuchel RP., Raftos DA., Birch D. and Vella N., 2010. Haemocyte morphology and function in the Akoya Pearl Oyster, Pinctada imbricata. Journal of Invertebrate Pathology, 105 (1): 0-48. https://doi.org/10.1016/j.jip.2010.04.011

Li J., Zhao N., Huang L., Zhou CJ. and Zhang YG., 2011. Structure and cytochemical characteristics of blood cells in myxocyprinus asiaticus. Acta Hydrobiologica Sinica, 35 (3): 550-556. https://doi.org/10.3724/SP.J.1035.2011.00550

Martina M., Lefevre SD. and Ostuni MA., 2017. From Erythroblasts to Mature Red Blood Cells: Organelle Clearance in Mammals. Frontiers in Physiology, 8:1076-1084. https://doi.org/10.3389/fphys.2017.01076

Murad A., Everill S. and Houston A., 1993. Division of goldfish erythrocytes in circulation. Canadian Journal of Zoology, 71 (11): 2190-2198. https://doi.org 10.1139/z93-308

Orci L. and Perrelet A, 1975. Blood. Springer Berlin Heidelberg, Switzerland.

Qing W., Yong-bo B., Zhi-hua L. and Hao-ming L., 2011. Comparison of erythrocytes of five aquatic animals and their evolution. Marine Sciences, 35 (10): 69-74.

Roger AJ. and Silberman JD, 2002. Cell evolution: Mitochondria in hiding. Nature, 418: 827-829. https://doi.org/10.1038/418827a

Rowley PT., 1995. Williams Hematology. J American Journal of Human Genetics. 
Ya Liu., Qing Xiao., Song Yang., Liulan Zhao., Hongmei Fu. and Jun Du., 2017. Characterization of hematopoiesis in Dabry's sturgeon (Acipenser dabryanus). Aquaculture and Fisheries, 2 (6): 262-268.

Zexia G., Weimin W., Yi Y., Abbas K. and Dapeng L., 2007. Morphological studies of peripheral blood cells of the Chinese sturgeon, Acipenser sinensis. Fish Physiology Biochemistry, 33 (3):213-222. https://doi.org/10.1007/s10695-007-9133-x 